\title{
COMPARATIVE ANALYSIS OF AUTOGRAFT TECHNIQUE AND CLAVICULAR HOOK PLATE IN GRADE 3 ACROMIOCLAVICULAR DISLOCATIONS
}

\author{
Rahul Narayan Bade1, Ojas Saini²
}

1 Professor, Department of Orthopaedics, Rajashree Chhatrapati Shahu Government Medical College, Kolhapur.

${ }^{2}$ Assistant Professor, Department of Orthopaedics, Rajashree Chhatrapati Shahu Government Medical College, Kolhapur.

\section{ABSTRACT}

\section{OBJECTIVE}

To evaluate clinical outcome of clavicular hook plate and autograft technique in the surgical treatment of Rockwood Grade-III acromioclavicular joint dislocation.

\section{METHODS}

Fifteen patients were operated using standard clavicular hook plate and another fifteen patients were operated using autograft technique. After a minimum follow-up of one year, clinical assessment was done using Constant-Murley Score. Statistical evaluation was done using SPSS ver. 21.0.

\section{RESULTS}

Mean age group was $31.44 \pm 7.8$ years. Average constant shoulder score for the hook plate group was 81.3 (Range 74-89) and that for autograft technique group was 91.7 (Range 88-97).

\section{CONCLUSION}

Autograft technique was found to be superior in terms of functional outcome when compared to clavicular hook plate. Such superiority can be attributed to the biologic nature of coracoclavicular ligament reconstruction in the autograft technique.

\section{KEYWORDS}

Acromioclavicular Dislocation; Autograft; Hook Plate.

HOW TO CITE THIS ARTICLE: Bade RN, Saini O. Comparative analysis of autograft technique and clavicular hook plate in grade 3 acromioclavicular dislocations. J. Evolution Med. Dent. Sci. 2016;5(34):1906-1908, DOI: 10.14260/jemds/2016/451

\section{INTRODUCTION}

Injuries to Acromioclavicular joint are fairly common and constitutes $12 \%$ of all injuries to shoulder girdle.(1) Acromioclavicular dislocation is classified as per criteria given by Rockwood et al.(2) In Grade III dislocation, both acromioclavicular and coracoclavicular ligaments are completely disrupted resulting in superior displacement of the distal clavicle. Many surgical treatments have been proposed to deal with such challenging injuries, but still there is no gold standard procedure yet.

Furthermore, conservative approach to Grade III Acromioclavicular dislocations is considered appropriate by many authors. (3) In this paper, we have done a comparative analysis between clavicular hook plate technique and autograft technique in a fairly young study population suffering from chronic Grade III Acromioclavicular dislocation.

\section{MATERIAL AND METHODS}

In our hospital, between May 2010 and February 2013, thirty patients who had Rockwood Grade III AC Dislocation were operated by the senior author.

Financial or Other, Competing Interest: None.

Submission 24-02-2016, Peer Review 06-04-2016,

Acceptance 11-04-2016, Published 27-04-2016.

Corresponding Author:

Dr. Rahul Narayan Bade,

142/5, Near Bus Stop,

Gandhinagar Road, Nigadewadi Taluk,

Karveer Dist.

Kolhapur-416005, Maharashtra.

E-mail:rbrahulbade@gmail.com

DOI: $10.14260 /$ jemds $/ 2016 / 451$
All the patents were operated under general anaesthesia in the beach chair position. Interscalene block was also given. In the first group of fifteen patients, clavicular hook plate was used to stabilize the acromioclavicular joint with no attempt at coracoclavicular reconstruction.

In the other group of remaining fifteen patients, tendon autograft in form of semitendinosus was harvested from ipsilateral knee using a tendon stripper. After standard graft preparation, drill holes were made in the distal clavicle corresponding to anatomical attachment points of coracoclavicular ligament and the graft was then passed under the coracoid process in a figure of eight fashion. After reduction of the $\mathrm{AC}$ joint, the graft ends were secured using Ethibond No. 5. In both the groups, distal clavicle excision as done in Mumford procedure, was performed in a standard fashion. Post-operatively, both the groups were placed in an universal shoulder immobilizer for two weeks till suture removal.

Pendulum exercises were started at post-operative day 15 and active range of motion was subsequently started. Strengthening protocol was begun after three months and patients were allowed to gradually return to their normal activities. Clinical assessment of recovery was made by an independent observer using Constant-Murley score. Such assessments were made after a mean follow-up of 15.3 months, \pm 3.8 months post-operatively. In this study, there were 24 males and 6 females with a mean age of $31.4 \pm 7.8$ years.

Statistical Analysis was made using SPSS ver. 21.0 (Chicago, USA) and rejection of null hypothesis was done when $\mathrm{p}$ values were $<0.05$. 
Student t-test, descriptive statistics and the MannWhitney U-test were employed to study outcome between the two treatment groups.

\section{OBSERVATIONS AND RESULTS}

Mean age of the entire study population was $31.4 \pm 7.8$ years. Average constant shoulder score for the hook plate group was 81.3 (Range 74-89) and that for autograft technique group was 91.7 (Range 88-97). This difference with regard to clinical outcome was found to be statistically significant. Furthermore, loss of reduction did not occur in the autograft technique group, but such failure was observed in three patients $(20 \%)$ of hook plate group. There was one incidence of superficial infection in autograft technique group and a single incidence of deep infection in hook plate group.

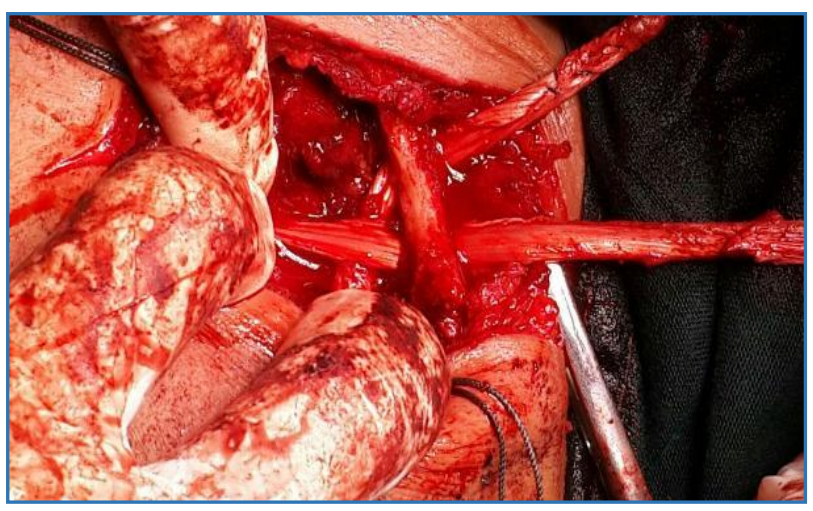

Fig. 1: Demonstration of Technique of Reconstruction of Coracoclavicular Ligament with Semitendinosus Graft

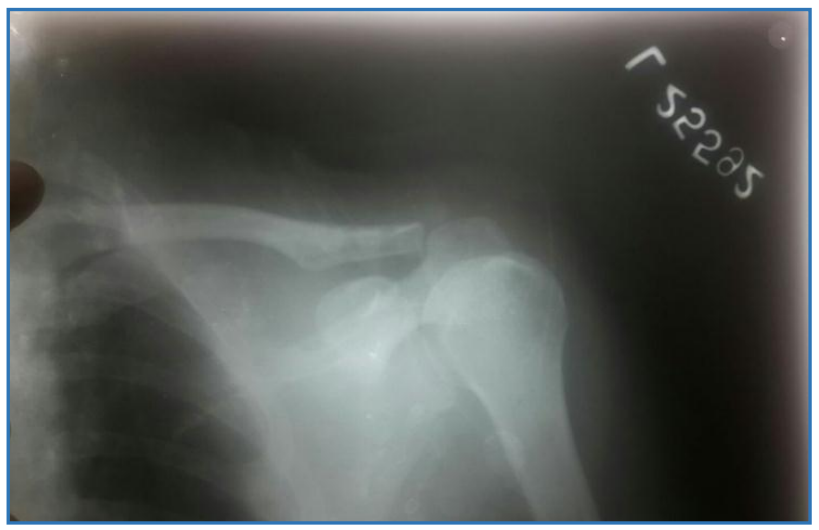

Fig. 2: Post-Op X-ray Depicting Clavicular Drill Holes for the Graft and Good Acromioclavicular Reduction

\section{DISCUSSION}

Acromioclavicular joint is a diarthrodial joint. Static stability is provided by coracoclavicular and acromioclavicular ligaments; whereas trapezius and deltoid contribute to dynamic stability of this joint. Acromioclavicular joint injuries are classified most commonly using the 6 grade system described by Rockwood.(2) Type I: Mild A.C. ligament sprain. Type II: Ruptured A.C. ligament with C.C. ligament sprain. Type III: Both C.C. and A.C. ligaments are ruptured. Clavicle elevated above the superior border of the acromion, but coracoclavicular distance is less than twice normal. Type IV: Clavicle displaced posteriorly into trapezius. Type V: Clavicle is markedly elevated and coracoclavicular distance is more than double normal (i.e. $>25 \mathrm{~mm}$ ). Type VI: Clavicle inferiorly displaced behind coracobrachialis and biceps tendons.

As far as treatment of acromioclavicular dislocations is concerned, there is quite a bit of confusion with regards to treating a Grade III injury. Despite our greater knowledge of tendon healing and incorporation and vast improvement in our surgical techniques, we are yet to arrive at a 'Gold Standard.' Grade III injuries are usually treated conservatively and various authors have obtained satisfactory results with conservative line of management. $(4,5)$ On the other hand, several reports have supported operative management of such injuries in young population who are physically active.(6,7) With regards to surgical options, variety of procedures have been described in the literature, viz. tension band wiring, K-wire fixation, hook plate, autograft or allograft reconstruction of acromioclavicular joint, Bosworth screw fixation, use of synthetic ligament, mersilene tape sling, etc. The purpose of our study was to compare outcome between clavicular hook plate and coracoclavicular ligament reconstruction using autograft in chronic Grade III injury. Hook plates have recently gained popularity, particularly over the traditional methods such as tension band wiring and K-wire fixation.

Traditional techniques had problems of metal breakage, pin migration, neurovascular injury, loss of reduction, implant failure, etc. With use of hook plates, such problems are not there. Several studies have published promising results with usage of hook plates. ${ }^{(8,9)}$ However, this technique is not without complications. Subacromial impingement is often reported; acromial erosions or distal clavicle fracture may occur due to altered biomechanics.(10,11) Loss of reduction may also occur, although it is much less common than traditional techniques. Second surgery for hardware removal is also advocated after complete healing.

Recent biomechanical studies have proved beyond doubt that reconstruction of acromioclavicular joint using tendon graft is more biological and the resultant repair tissue is desirable in terms of strength and kinematics.(12) Most of the described reconstruction techniques deal only with coracoclavicular ligament, but it has been shown that additional acromioclavicular ligament reconstruction adds to the final stability in a significant way. $(13,14,15)$ However, in our study we aimed to reconstruct coracoclavicular ligament only. As per our results, we observed a significant difference between clinical outcome of graft reconstruction versus hook plate fixation. Anatomic technique using semitendinosus graft showed improved outcome over the non-anatomic procedure using hook plate. Similar results have been reproduced by various authors as well, in both clinical and cadaveric scenarios. $(16,17,18)$ This is in accordance with idea of anatomic reconstruction of damaged ligaments after they have been injured.

Various procedures around the knee now focus on defining isometric points and subsequent anatomic reconstruction. Hook plates have an inherent disadvantage due to its non-biologic nature leading to stress shielding effect and subacromial impingement. Furthermore, they invariably require second surgery for hardware removal. Considering such disadvantages, we would to like to recommend autograft technique over hook plates in chronic Grade III acromioclavicular injuries. Our study has few limitations. The study population was relatively small. Minor modifications in surgical technique might have occurred with passage of time 
as reconstruction of coracoclavicular ligament was a demanding procedure and we were relatively new to this technique. Still we tried our best not to allow any major change in the operative technique, so as to keep such a bias to minimum in our study.

\section{CONCLUSION}

In conclusion, we obtained favourable results in autograft technique of reconstruction of coracoclavicular ligament when compared to A.C. joint fixation with hook plate in chronic Grade III A.C. dislocations. We attribute this to the biological nature of anatomic reconstruction of A.C. joint. Furthermore, hook plates have additional problems which include subacromial impingement, acromial erosions, distal clavicle fractures, need for second surgery for hardware removal, etc.

\section{ACKNOWLEDGEMENT}

All the authors read and approved the final manuscript. No conflicts of interest were declared by the authors. No external funding was received for this study.

\section{REFERENCES}

1. Emery R. Acromioclavicular and sternoclavicular joints. In: Copeland S, editor. Shoulder surgery. WB Saunders; London: 1997.

2. Rockwood C, Williams G, Young D. Disorders of the acromioclavicular joint. In: Rockwood CJ, Matsen FA, editors. The Shoulder. WB Saunders; Philadelphia, 1998; $2^{\text {nd }}$ edition:483-553.

3. Faldini C, Nanni M, Leonetti D, et al. Nonoperative treatment of closed displaced midshaft clavicle fractures. J Orthop Traumatol 2010;11(4):229-36.

4. Fremerey RW, Lobenhoffer P, Ramacker K, et al. Acute acromioclavicular joint dislocation-operative or conservative therapy? Unfallchirurg 2001;104(4):294-9.

5. Gstettner C, Tauber M, Hitzl W, et al. Rockwood type III acromioclavicular dislocation: surgical versus conservative treatment. J Shoulder Elbow Surg 2008;17(2):220-5.

6. Glick JM, Milburn LJ, Haggerty JF, et al. Dislocated acromioclavicular joint: follow- up study of 35 unreduced acromioclavicular dislocations. Am J Sports Med 1977;5:264-70.
7. Rawes ML, Dias JJ. Long-term results of conservative treatment for acromioclavicular dislocation. J Bone Joint Surg Br 1996;78:410-2.

8. Salem KH, Schmelz A. Treatment of tossy III acromioclavicular joint injuries using hook plates and ligament suture. J Orthop Trauma 2009;23(8):565-9.

9. Von Heideken J, Bostrom Windhamre H, Une-Larsson V, et al. Acute surgical treatment of acromioclavicular dislocation type $\mathrm{V}$ with a hook plate: superiority to late reconstruction. J Shoulder Elbow Surg 2013;22(1):9-17.

10. Chiang CL, Yang SW, Tsai MY, et al. Acromion osteolysis and fracture after hook plate fixation for acromioclavicular joint dislocation: a case report. J Shoulder Elbow Surg 2010;19(4):e13-15.

11. Hoffler CE, Karas SG. Transacromial erosion of a locked subacromial hook plate: case report and review of literature. J Shoulder Elbow Surg 2010;19(3):e12-15.

12. Costic RS, Labriola JE, Rodosky MW, et al. Biomechanical rationale for development of anatomical reconstructions of coracoclavicular ligaments after complete acromioclavicular joint dislocations. Am J Sports Med 2004;32(8):1929-36.

13. Jari R, Costic RS, Rodosky MW, et al. Biomechanical function of surgical procedures for acromioclavicular joint dislocations. Arthroscopy 2004;20(3):237-45.

14. Harris RI, Wallace AL, Harper GD, et al. Structural properties of the intact and the reconstructed coracoclavicular ligament complex. Am J Sports Med 2000;28(1):103-8.

15. Beitzel K, Cote MP, Apostolakos J, et al. Current concepts in the treatment of acromioclavicular joint dislocations. Arthroscopy 2013;29(2):387-97.

16. Grutter PW, Petersen SA. Anatomical acromioclavicular ligament reconstruction: a biomechanical comparison of reconstructive techniques of the acromioclavicular joint. Am J Sports Med 2005;33(11):1723-8.

17. Thomas K, Litsky A, Jones G, et al. Biomechanical comparison of coracoclavicular reconstructive techniques. Am J Sports Med 2011;39(4):804-10.

18. Lee SJ, Keefer EP, McHugh MP, et al. Cyclical loading of coracoclavicular ligament reconstructions: a comparative biomechanical study. Am J Sports Med 2008;36(10):1990-7. 